\title{
Factors Influencing Utilization of Ultrasound Scan Services among Pregnant Mothers at Ndejje Health Center IV, Wakiso District . \\ journal of gynecology and obstetrics@SJHR-Africa
}

\author{
Angella Nakimera ${ }^{a, 1,2}$ \\ a Department of Midwifery, Nsambya School of Nursing and Midwifery, \\ Nsambya, Kampala, Uganda
}

\begin{abstract}
Background: A study was carried out to identify the factors influencing the utilization of ultrasound scan services among pregnant mothers at Ndejje Health Centre IV, Wakiso District.

Methodology: The study design was descriptive and cross-sectional and it employed both quantitative and qualitative data collection methods. A sample size of 30 respondents was selected using a simple random sampling procedure. An interview guide was used to collect data.

Results: The study revealed various socio-economic factors influencing the utilization of ultrasound scan services among pregnant mothers. For example, although all $30(100 \%)$ had ever heard about ultrasound scan services, most 20 (66.7\%) had ever used ultrasound scan services once $10(50 \%)$ and 12 (60\%) used the services in the 3rd trimester due to factors including 20 (66.7\%) unaffordability of ultrasound scan services, 21 (70\%) having fears about using ultrasound scan services including 14 (66.7\%) fear that the scan would identify bad conditions on the baby, $18(60 \%)$ lacked partner support. The study results also revealed that respondents faced various health facility factors which influenced the utilization of ultrasound scan services. For example, most 20 (66.7\%) respondents reported that Ndejje Health Centre IV was not equipped to provide ultrasound scan services due to 12 (60\%) frequent breakdown and poor maintenance of equipment which led to $18(60 \%)$ ultrasound scan services not being readily available, $12(60 \%)$ long waiting time to receive services as most waited more than 2 hours to receive services.

Conclusion and recommendations: Respondents faced various socio-economic and health facilityrelated factors which influenced their utilization of ultrasound scan services. The key recommendations included ready availability of services through regular and timely maintenance of equipment, improving efficiency, and reducing waiting time as well as improved health education of mothers about the importance of using the services.
\end{abstract}

\section{Background:}

The use of ultrasound scan services by pregnant women remains a challenge globally (Papageorghiou et al., 2016). An ultrasound scan is a diagnostic imaging technique based on the application of ultrasound waves (Papageorghiou et al., 2016). It is used to create an image of internal body structures and organs and its aim was often to find a source of a disease or to exclude pathology (Abdullah, 2019).

\footnotetext{
${ }^{1}$ Corresponding author.

2E-mail: nakimeraek@gmail.com
} 
Ultrasound has been used in obstetrics since the 1950s and has become an essential part of current prenatal care (Mbuyita et al., 2015). The use of ultrasound in pregnancy provided valuable information that could predict fetal outcomes and aid in the detection of cardiac, gastrointestinal, renal, and neural abnormalities as well as chromosomal anomalies including Down's syndrome(Mcclure et al., 2017).

Routine ultrasound in pregnancy is useful for the determination of multiple pregnancies and gestational age and may be associated with a lower likelihood of inductions after 42 weeks (Anna et al.,2016). Current recommendations by the Society of Obstetricians and Gynecologists of Canada (SOGC) stated that all pregnant women should be offered an ultrasound scan between 18 and 22 weeks to screen for fetal anomalies and to provide information about the placenta, gestational age, and a number of foetuses.

According to Abdullah et al., (2016) Besides, the SOGC recommended that all pregnant patients be offered an ultrasound scan between 11 and 14 weeks of pregnancy to confirm gestational age and viability as well as investigate the number of fetuses, early anatomical assessment, and nuchal translucency. However, its use by women was often influenced by various socio-economic, health worker, and health facility factors.

Globally, the use of ultrasound scans by pregnant women varied greatly from one country to another. For instance, in rural eastern China,(Huang et al., 2012), found very high use of antenatal ultrasound, and this was influenced by various socio-demographic and clinical factors such as availability of support, availability, and accessibility of services as well as improved access to information among others. However, in countries like Venezuela, Whitworth, Bricker, and Mullan, (2015) found low use of ultrasound scan services due to factors such as high costs, poor service coverage as well as lack of awareness about its benefits.

In Sub Saharan Africa, the use of ultrasound scans by pregnant women also varies. For example, in Lagos State, Nigeria, Ugwu, Elugwu, and Onyegbule (2016) found increased use of ultrasound scans by pregnant women. This was attributed to factors such as the education level of the couple, especially the head of the household, improved socio-economic status among other factors while in Kano, Northern Nigeria, there was less utilization of ultrasound scans due to poor coverage of services and lower incomes among other factors.

In East Africa, the situation remained similar to the above as exemplified in a study by Oluoch et al., (2015), in Nairobi Kenya whose study revealed that the use of ultrasound scans by pregnant women was influenced by women's awareness of the services and its benefits, availability of services, customer care, availability of support as well as the quality of services provided among other factors.

In Uganda, the use of ultrasound scan services varied and according to Swanson et al.,(2014), the use of ultrasound scan services by pregnant women was more predominant in cities and urban centres as compared to rural areas. This was generally attributed to factors such as poverty, level of education, long distance to health facilities, unavailability of services, perceived low quality of services, and lack of support among others.

Poor uptake and utilization of ultrasound scans among pregnant women lead to many poor outcomes such as maternal and neonatal deaths, yet antenatal ultrasound scan is a widely accepted component of antenatal care and it could be used to detect a pregnancy as early as 5 weeks(Becker et al., 2016). Furthermore, pregnant women who did not use ultrasound scans were more predisposed to conditions such as uncertain menstrual dates, abnormal pregnancies (e.g. ectopic, molar, etc.), and pelvic masses(Swanson, 2017).

At Ndejje Health Centre IV, Wakiso District, ultrasound scan services were provided to pregnant women. However, according to Health Management Information System (HMIS) records at the health centre, in Financial Year (FY) (2016/2017), 78\% of all pregnant women that attended ANC services did not use ultrasound scans, in 2017/2018, 83\% of pregnant women did not use ultrasound scans while in 2018/2019, $89 \%$ of pregnant women did not use ultrasound scans despite the availability of the service. However, the particular factors influencing the use of ultrasound scans by pregnant women were unknown as no study had been carried out to identify them. It was hence according to this background that the researcher picked interest to carry out this study and identify the factors influencing utilization of ultrasound scan services among pregnant mothers at Ndejje Health Centre IV. 


\section{Study Design and Rationale}

\section{Methodology}

The study was descriptive and cross-sectional employing both qualitative and quantitative data collection methods. This study design was selected because it helped to get data at a single point.

\section{Study Setting and Rationale}

The study was conducted at Ndejje Health Centre IV, in Ndejje Zanta Village in Wakiso District which is found in the Central Region of Uganda. The health centre is located $10.4 \mathrm{~km}$ from Kampala City, Shoprite Clock Tower along Entebbe road to Ndejje Health Centre IV (6km along Entebbe road and $4.4 \mathrm{~km}$ off Entebbe Road to Ndejje road up to the Health Centre) and its latitude is: 0.6060 and its longitude is 32: 4717. The health facility offers many health care services including ANC, Ultrasound scan services, child health services, HIV/AIDS management services, general patient management, laboratory services, nutrition services, antenatal and postnatal services among many others. The study setting was selected because it was well known to the researcher and the problem of poor uptake of ultrasound scan services had been noted on the ground.

\section{Study Population}

The study included pregnant mothers attending health care services at Ndejje Health Centre IV, Wakiso District. The study population was 60 respondents.

\section{Sample Size}

The sample size was 30 respondents, all pregnant mothers attending health care services at Ndejje Health Centre IV, Wakiso District.

\section{Sampling Procedure}

The researcher utilized a simple random sampling procedure to select respondents to participate in the study. In this study, the researcher wrote the words YES and NO on 60 pieces of paper, 30 with YES and 30 with NO written on them, folded and put in an enclosed box, shook it, and then invited respondents to participate by randomly selecting a piece of paper from the box. Any respondent who picked a paper with the word YES written on it was requested to participate in the study. This continued until the total number of respondents to be interviewed per day was achieved.

\section{Inclusion Criteria}

The study included only pregnant mothers attending health care services at Ndejje Health Centre IV who were available at ANC clinic during the data collection days and were willing to voluntarily consent to participate in the study. The study excluded all mothers who refused to consent to participate in the study.

\section{Definition of Variables}

The dependent variables for the study included:

Utilization of ultrasound scan services

The independent variables for the study included:

Socio-economic factors

Health facility factors

\section{Research Instruments}

Data was collected using an approved semi-structured interview guide which consisted of both open and closed-ended questions. This tool was selected because the study involved mixed respondents who were unable to read, write, and understand English used to develop the questionnaire.

\section{Data Collection Procedure}

Before approaching and collecting data from respondents at the health centre, the researcher was accompanied and introduced to the respondents by the in-charge of the ANC clinic. The researcher administered interview guides to mothers at the ANC clinic or from the health centre grounds. This improved efficiency and confidentiality during data collection. The researcher sampled 10 respondents per day for a total of 30 respondents for 3 days.

\section{Data Management}


Data management included data editing before leaving the area of study to ensure that there were no mistakes or areas left blank, and any found were corrected before leaving the area of study.

\section{Data Analysis and Presentation}

The collected data was first analysed manually by the use of papers and pens and tallying, after which the researcher presented them in tables, graphs, and pie charts generated by Microsoft Excel.

\section{Pilot Study}

The interview guide was pretested among 6 pregnant mothers attending health care services at Kajjansi Health Centre IV to enable the researcher to assess its clarity, accuracy, and reliability and made the necessary adjustments.

\section{Ethical Considerations}

A letter of introduction was obtained from St. Francis Hospital Nsambya Training School introducing the researcher to the administration of Ndejje Health Centre IV and seeking permission to carry out the study. After permission was granted, the overall in-charge introduced the researcher to the in-charge of the ANC clinic who in turn introduced the researcher to the respondents. Respondents were assured of maximum confidentiality and only numbers instead of names were used to identify the respondents. The study only commenced after the objectives of the study had been well explained to participants and they had consented to participate in the study.

\section{Data Analysis and Presentation 5 Demographic and Social Characteristics}

Figure 1: Respondents by age $n=30$

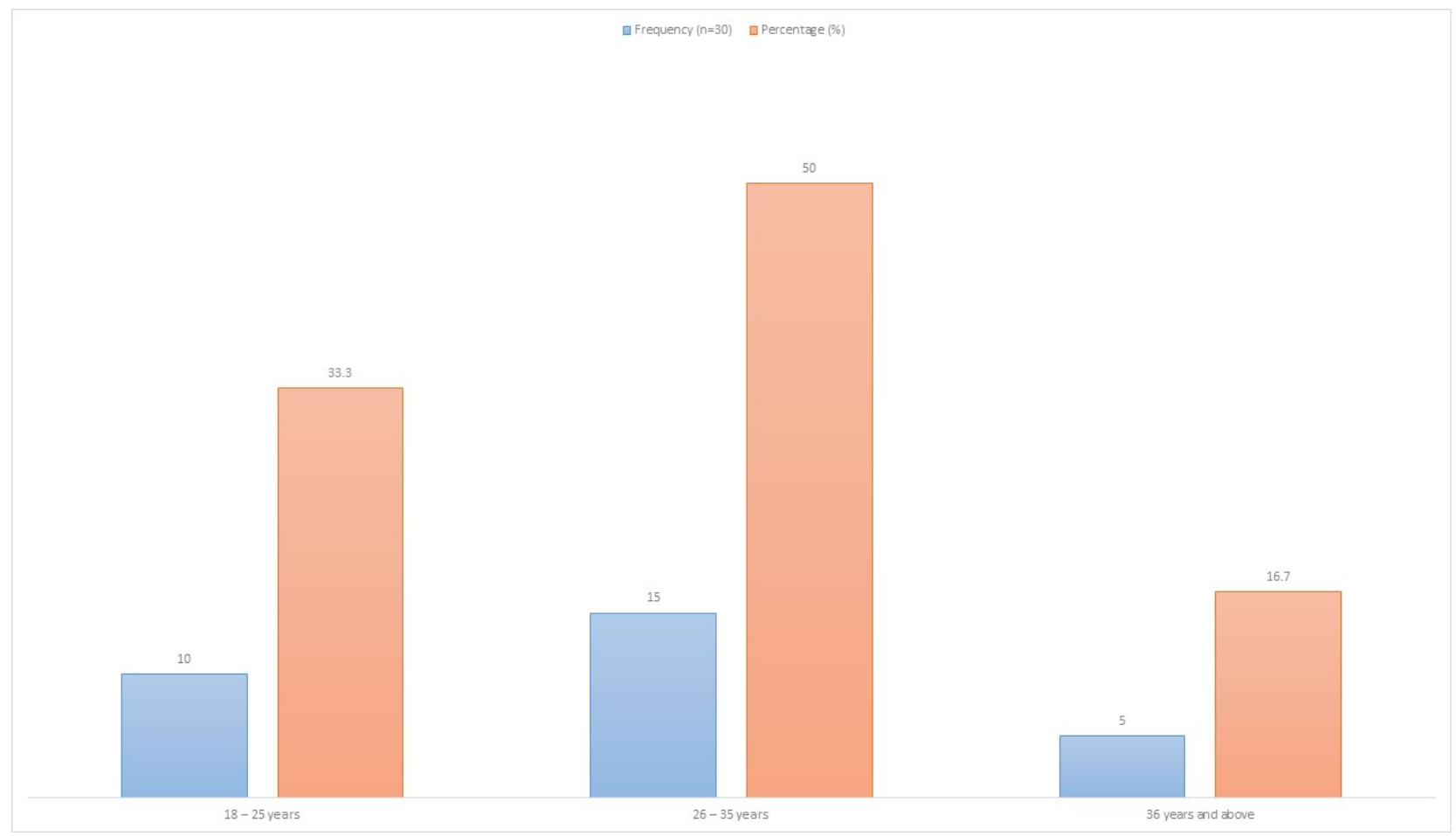

Chart 1. Respondents by age

Half $15(50 \%)$ of the respondents were in the age range of 26 - 35 years, followed by $10(33.3 \%)$ were in the age range of $18-25$ years while the least $5(16.7 \%)$ were 36 years and above. 


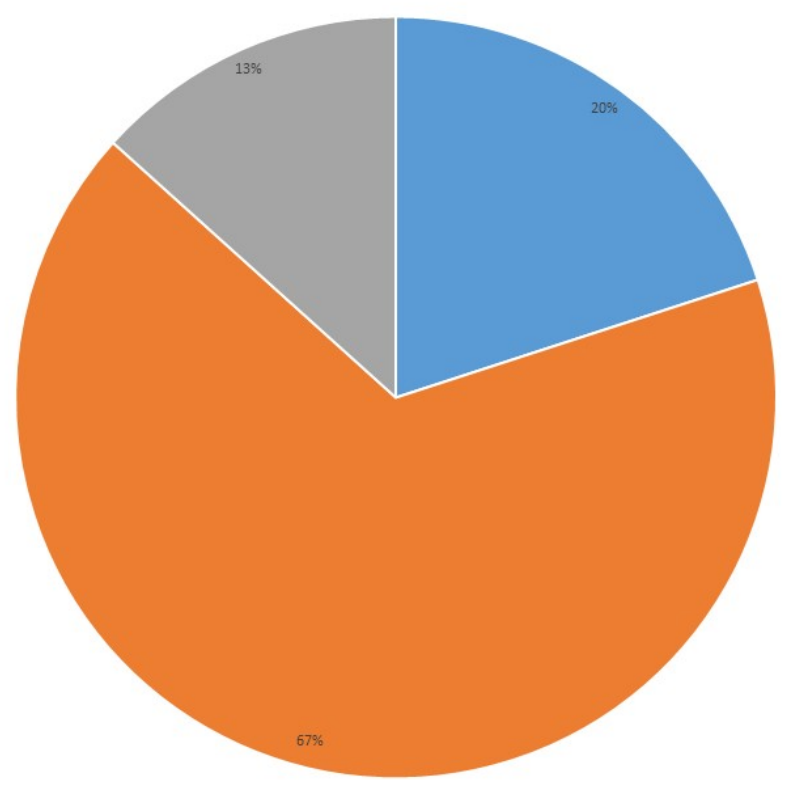

Chart 2. Respondents by marital

Table 1. Respondents by level of education

\begin{tabular}{lll}
\hline Level of education & Frequency (f) & Percentage (\%) \\
No formal education & 4 & 13.3 \\
Primary level & 6 & 20 \\
Secondary level & 12 & 40 \\
Tertiary level & 8 & 26.7 \\
Total & $\mathbf{3 0}$ & $\mathbf{1 0 0}$ \\
\hline
\end{tabular}

\section{Figure2: Respondents by marital status $n=30$}

Most $20(66.7 \%)$ respondents were married, followed by $6(20 \%)$ who were single while the least $4(13.3 \%)$ were widowed.

\section{Table 1: Respondents by level of education $\quad n=30$}

Results showed that $12(40 \%)$ respondents attained secondary level education, 8 (26.7\%) who attained tertiary level education, $6(20 \%)$ attained primary level education while the least 4 (13.3\%) did not attain any formal education.

\section{Figure3: Respondents by occupation $\mathrm{n}=\mathbf{3 0}$}

Results showed that 12 (40\%) respondents were self-employed, followed by 8 (26.7\%) who were professionals, 6 (20\%) were house wives while the least 4 (13.3\%) were peasants.

\section{Table 2: Respondents by level of education of spouse $\quad \mathbf{n}=\mathbf{2 0}$}

Out of the 20 married respondents, most 8 (40\%) attained tertiary level education, followed by 5 (25\%) attained secondary level education, 4 (20\%) attained primary level education while the least $3(15 \%)$ did not attain any formal education. 


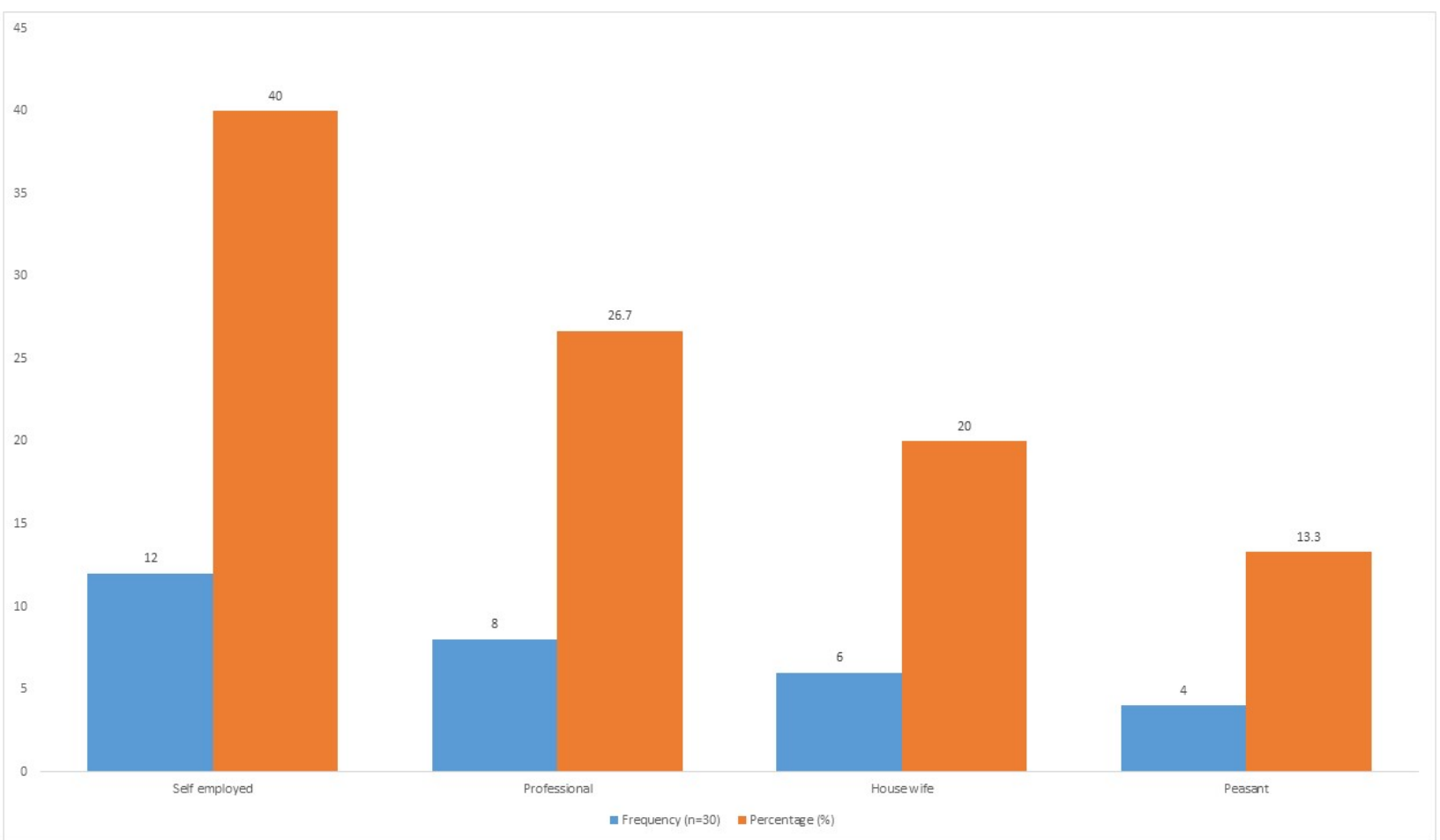

Chart 3. Respondents by occupation

Table 2. Respondents by level of education of spouse

\begin{tabular}{lll}
\hline Level of education & Frequency(f) & Percentage (\%) \\
No formal education & 3 & 15 \\
Primary level & 4 & 20 \\
Secondary level & 5 & 25 \\
Tertiary level & 8 & 40 \\
Total & $\mathbf{2 0}$ & $\mathbf{1 0 0}$ \\
\hline
\end{tabular}

10 Table 3: Respondents by occupation of spouse $n=20$

Out of the 20 married respondents, most 7 (35\%) respondents' spouses were self-employed, followed by 6 (30\%) who were professional, 5 (25\%) were peasants while the least $2(10 \%)$ were unemployed.

\section{Figure4: Respondents by parity $n=30$}

Half 15 (50\%) of the respondents reported having a parity of $2-3$ pregnancies, followed by 10 (33.3\%) who reported a parity of more than 4 pregnancies while the least $5(16.7 \%)$ had a parity of 1 pregnancy.

Table 3. Respondents by occupation of spouse

\begin{tabular}{lll}
\hline Occupation & Frequency(f) & Percentage (\%) \\
Self employed & 7 & 35 \\
Professional & 6 & 30 \\
Peasant & 5 & 25 \\
Unemployed & 2 & 10 \\
Total & $\mathbf{2 0}$ & $\mathbf{1 0 0}$ \\
\hline
\end{tabular}




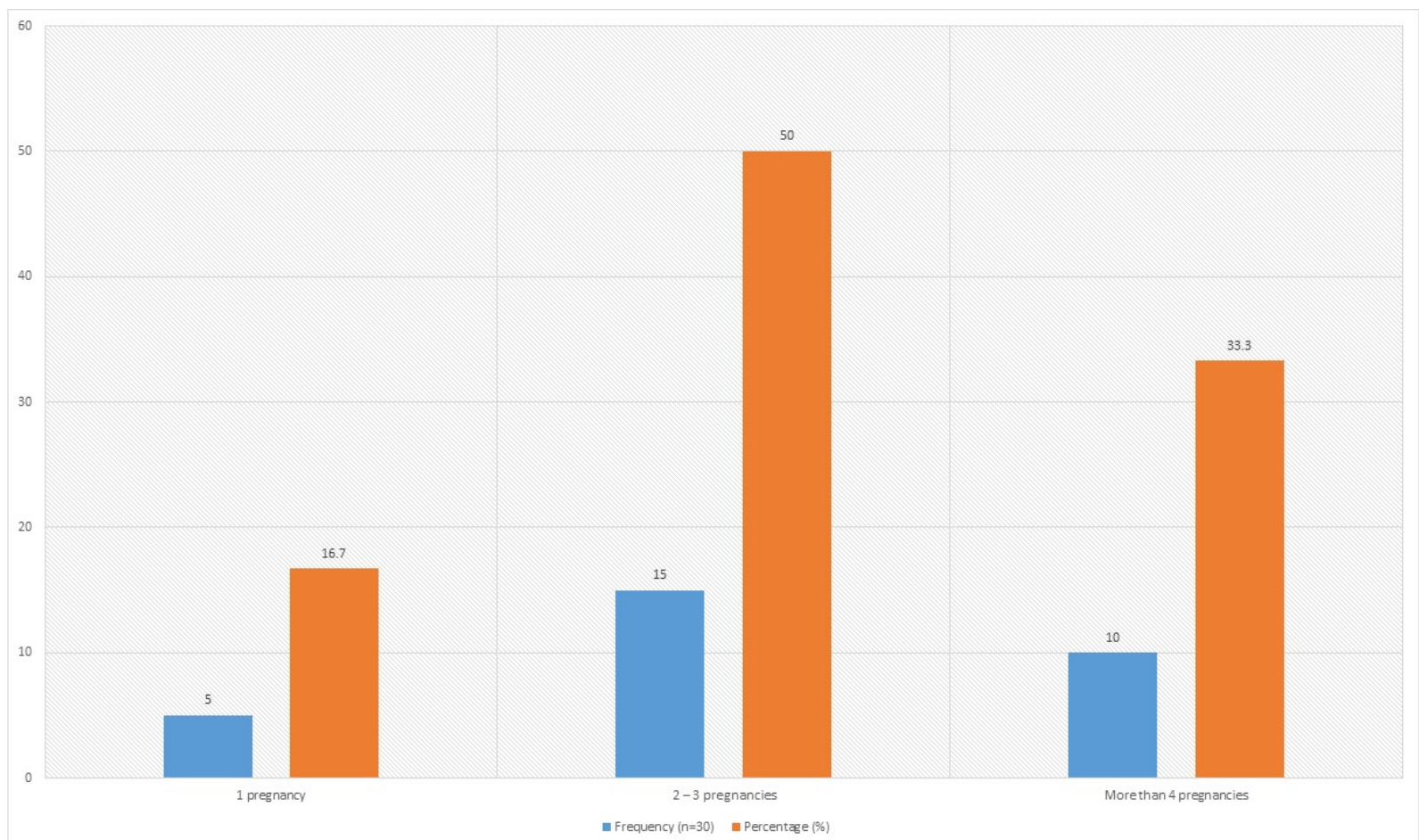

Chart 4. Respondents by parity

Table 4. Ever heard about ultrasound scan services

\begin{tabular}{lll}
\hline Responses & Frequency(f) & Percentage (\%) \\
Yes & 30 & 100 \\
No & 0 & 0 \\
Total & $\mathbf{3 0}$ & $\mathbf{1 0 0}$ \\
\hline
\end{tabular}

11.1 Socio Economic Factors Influencing Utilization of Ultrasound Scan Services Among Pregnant Mothers

12 Table 4: Ever heard about ultrasound scan services $\quad n=30$ All 30 (100\%) had ever heard about ultrasound scan services.

\section{Figure5: Source of information about ultrasound scan services $\mathrm{n}=\mathbf{3 0}$}

Most 20 (66.7\%) respondents obtained information about ultrasound scan services from health workers, followed by 6 (20\%) who obtained information from family and friends while the least $4(13.3 \%)$ obtained information from mass media.

\section{Table 5: Ever been health educated about the benefits of using ultrasound scan services $\mathrm{n}=30$}

Majority 20 (66.7\%) of respondents had ever been health educated about the benefits of using ultrasound scan services while the least 10 (33.3\%) had never been health educated. 


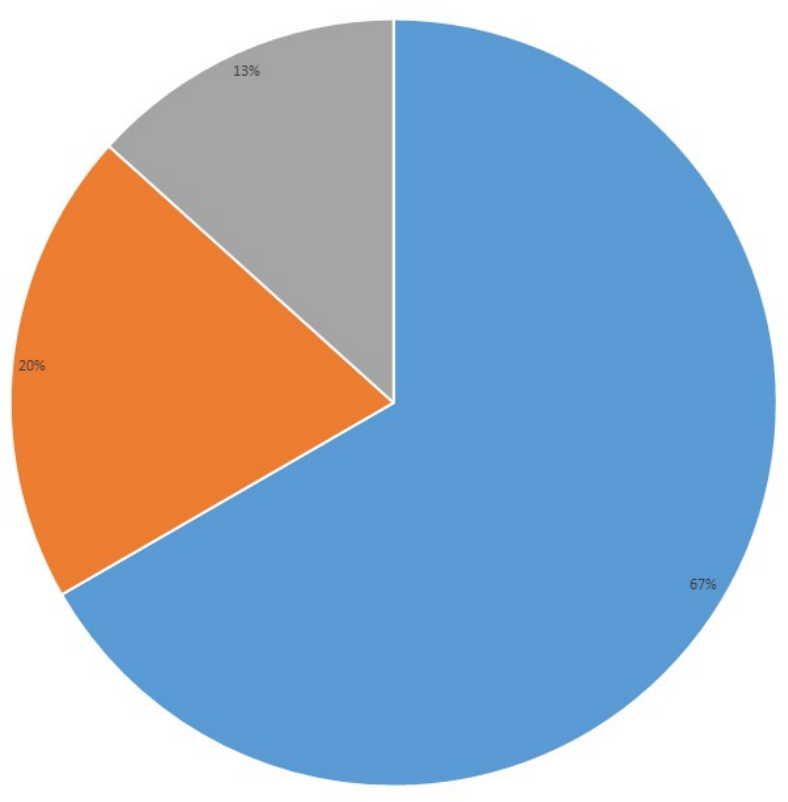

Chart 5. Source of information about ultrasound scan services

Table 5. Ever been health educated about the benefits of using ultrasound scan services

\begin{tabular}{lll}
\hline Responses & Frequency(f) & Percentage (\%) \\
Yes & 20 & 66.7 \\
No & 10 & 33.3 \\
Total & $\mathbf{3 0}$ & $\mathbf{1 0 0}$ \\
\hline
\end{tabular}

\section{Table 6: Benefits of using ultrasound scan services during pregnancy $n=20$}

Out of the 20 respondents who had ever been health educated about the benefits of using ultrasound, most $12(60 \%)$ reported that it helped to assess the health of the foetus while the least $8(40 \%)$ reported that it helped to detect any challenges on the foetus.

\section{Figure6: Whether respondents had ever used ultrasound scan services $\mathrm{n}=30$}

Majority 20 (66.7\%) of respondents had ever used ultrasound scan services while the least 10 (40\%) had never used ultrasound scan services.

Table 6. Benefits of using ultrasound scan services during pregnancy

\begin{tabular}{lll}
\hline Benefits & Frequency(f) & Percentage (\%) \\
Helps to assess the health of the foetus & 12 & 60 \\
Helps to detect any challenges on the foetus & 8 & 40 \\
Total & $\mathbf{2 0}$ & $\mathbf{1 0 0}$ \\
\hline
\end{tabular}




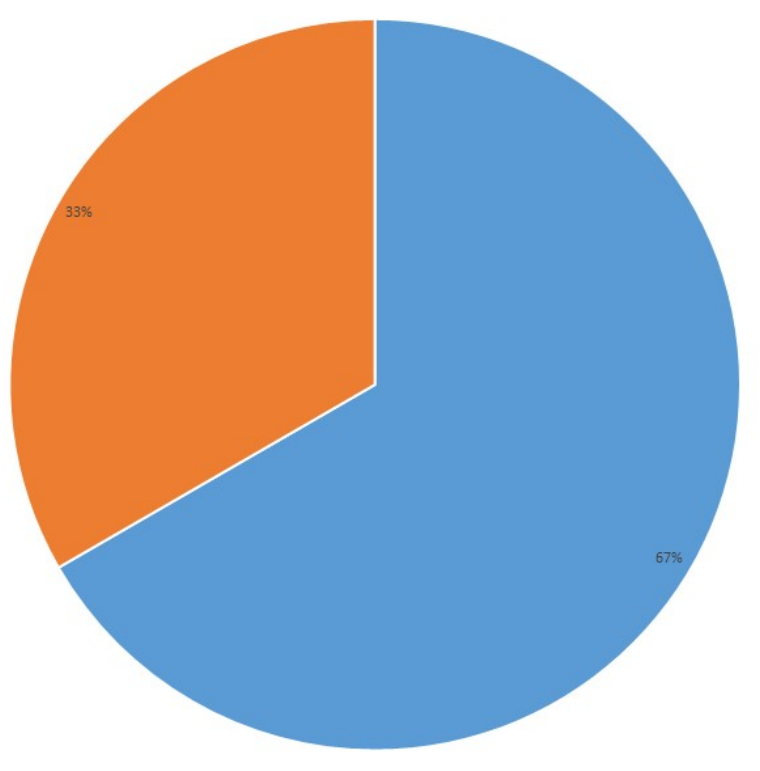

Chart 6. shows whether respondents had ever used ultrasound scan services

Table 7. Number of times respondents had used ultrasound scan services

\begin{tabular}{lll}
\hline Number of times & Frequency(f) & Percentage (\%) \\
Once & 10 & 50 \\
Twice & 7 & 35 \\
More than twice & 3 & 15 \\
Total & $\mathbf{2 0}$ & $\mathbf{1 0 0}$ \\
\hline
\end{tabular}

\section{Table 7: Number of times respondents had used ultrasound} scan services $n=20$

Most respondents 10 (50\%) had used the ultrasound scan services once, followed by 7 (35\%) who used the services twice while the least $3(15 \%)$ had used ultrasound scan services more than twice.

\section{Table 8: Rating of the cost of ultrasound scan services}

$$
\mathrm{n}=\mathbf{2 0}
$$

Out of the 20 respondents who had used ultra sound scan services, most 15 (75\%) rated the cost of ultrasound scan services as high while the least $5(25 \%)$ rated it as low.

Table 8. Rating of the cost of ultrasound scan services

\begin{tabular}{lll}
\hline Rating of cost & Frequency(f) & Percentage (\%) \\
High & 15 & 75 \\
Low & 5 & 25 \\
Total & $\mathbf{2 0}$ & $\mathbf{1 0 0}$ \\
\hline
\end{tabular}


19 Figure7: Whether the cost of ultrasound scan services was affordable $n=30$

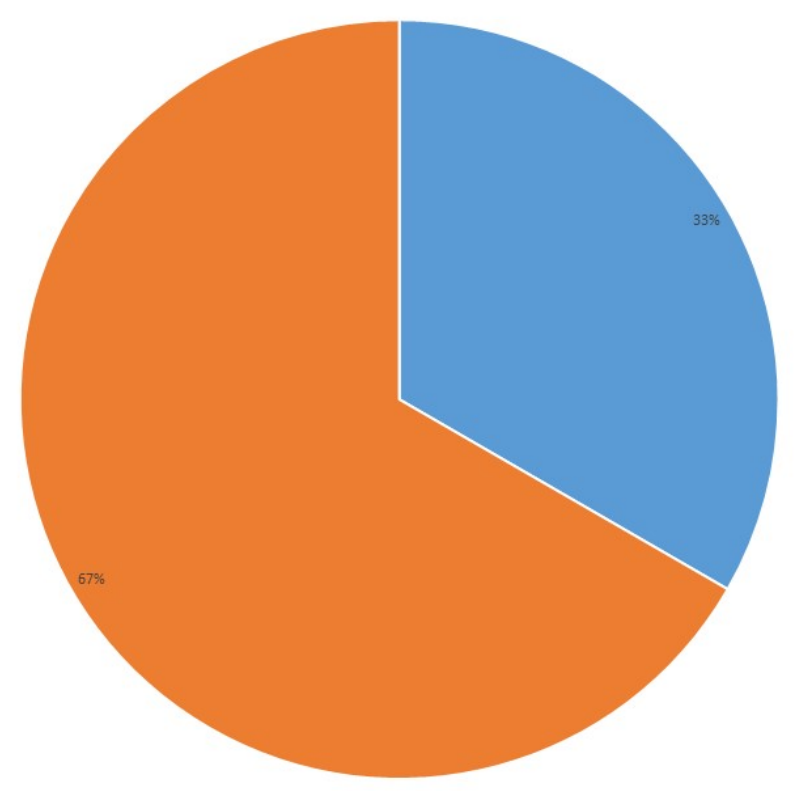

Chart 7. Whether the cost of ultrasound scan services was affordable

Most $20(66.7 \%)$ respondents reported that the cost of ultrasound scan services was not affordable while the least 10 (33.3\%) reported that it was affordable.

\section{Figure8: Whether unaffordability of ultrasound scan services affected utilization of services $\quad n=30$}

Majority 25 (83.3\%) of respondents agreed that unaffordability of ultrasound scan services affected utilization of services while the least 5 (16.7\%) disagreed.

\section{Figure9: Trimester in which respondents used ultrasound scan services $\mathbf{n}=\mathbf{2 0}$}

Most 12 (60\%) used the services in the $3^{\text {rd }}$ trimester, followed by $5(25 \%)$ who used the services in the $2^{\text {nd }}$ trimester while the least $3(15 \%)$ used the services in $1^{\text {st }}$ trimester.

\section{Figure 10: Whether respondents had any fears about using ultrasound scan services $\mathrm{n}=\mathbf{3 0}$}

Majority 21 (70\%) of respondents reported having fears about using ultrasound scan services while the least 9 (30\%) did not have any fears about using the ultrasound scan services.

\section{Table 9: Fears about using ultrasound scan services}

$$
\mathrm{n}=\mathbf{2 1}
$$

Most 14 (66.7\%) reported fears that the scan will identify bad conditions on the baby while the least 7 (33.3\%) reported fears about what it will reveal. 
Chart 8. Whether unaffordability of ultrasound scan services affected utilization of services

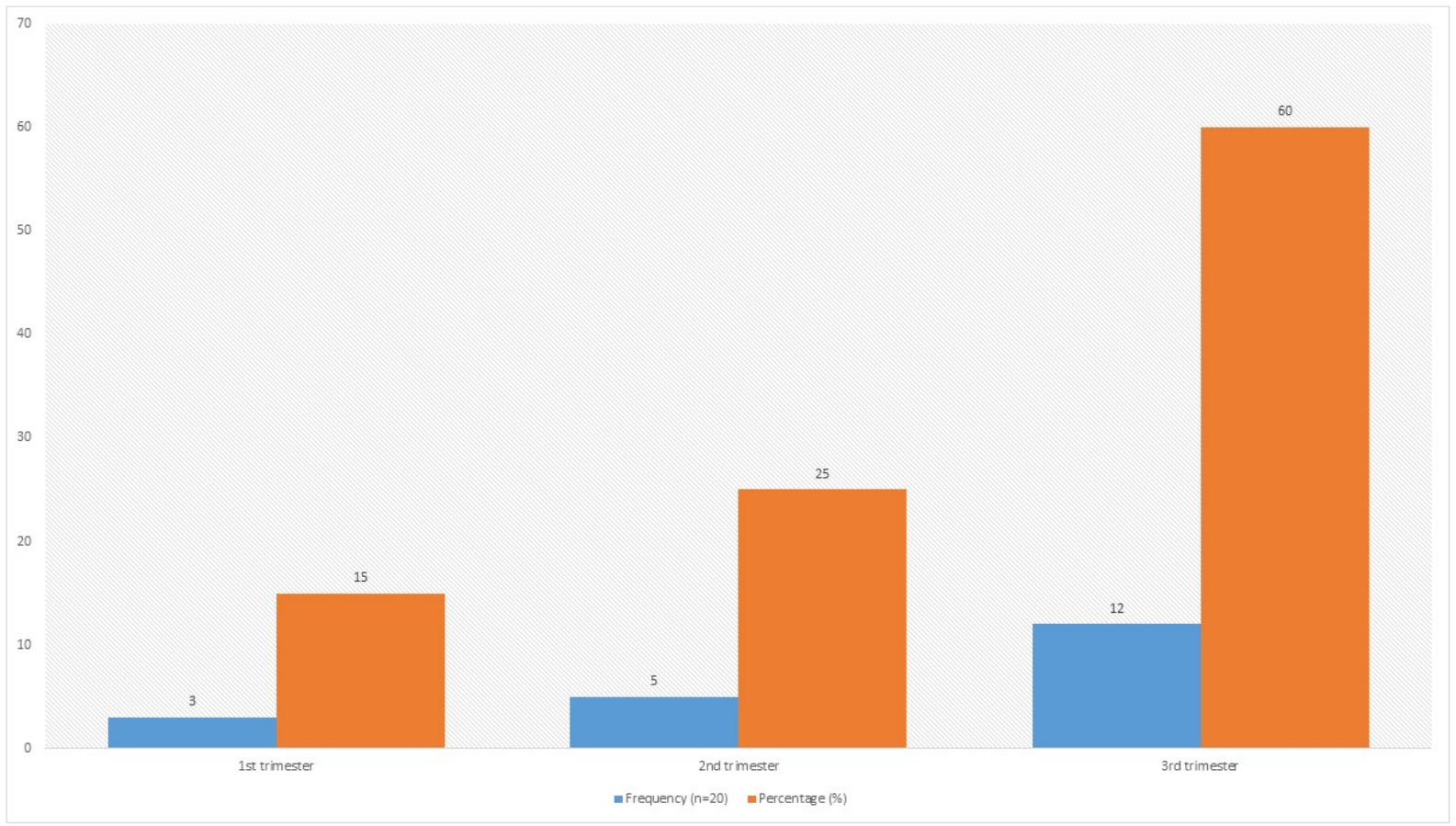

Chart 9. Trimester in which respondents used ultrasound scan services

Table 9. Fears about using ultrasound scan services

\begin{tabular}{lll}
\hline Fears & Frequency(f) & Percentage (\%) \\
Fear about what it will reveal & 7 & 33.3 \\
Fears that it will identify bad conditions on the baby & 14 & 66.7 \\
Total & $\mathbf{2 1}$ & $\mathbf{1 0 0}$ \\
\hline
\end{tabular}




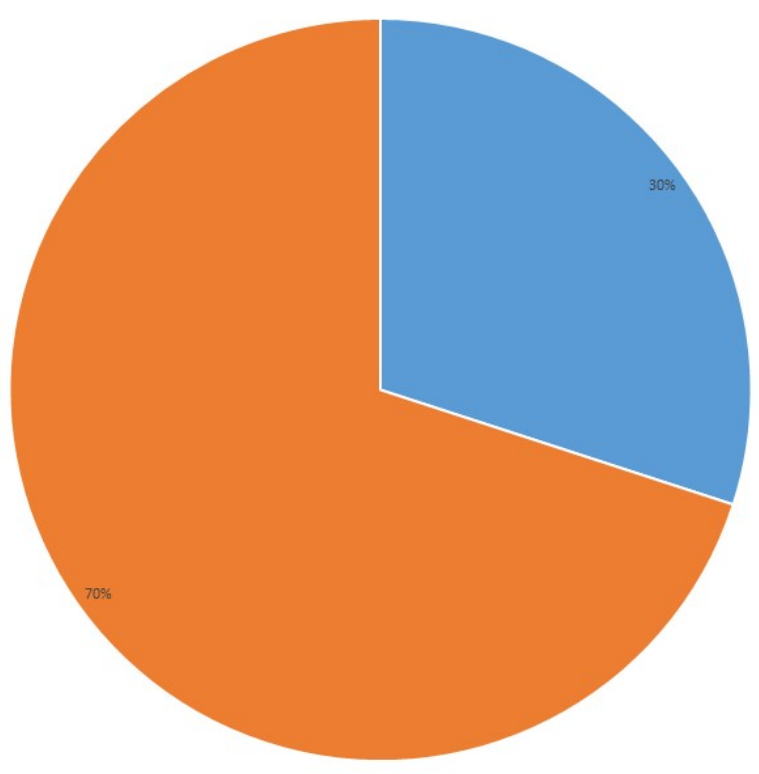

Chart 10. Whether respondents had any fears about using ultrasound scan services

\section{Figure 11: Whether fears can stop respondents from using ultrasound scan services $n=21$}

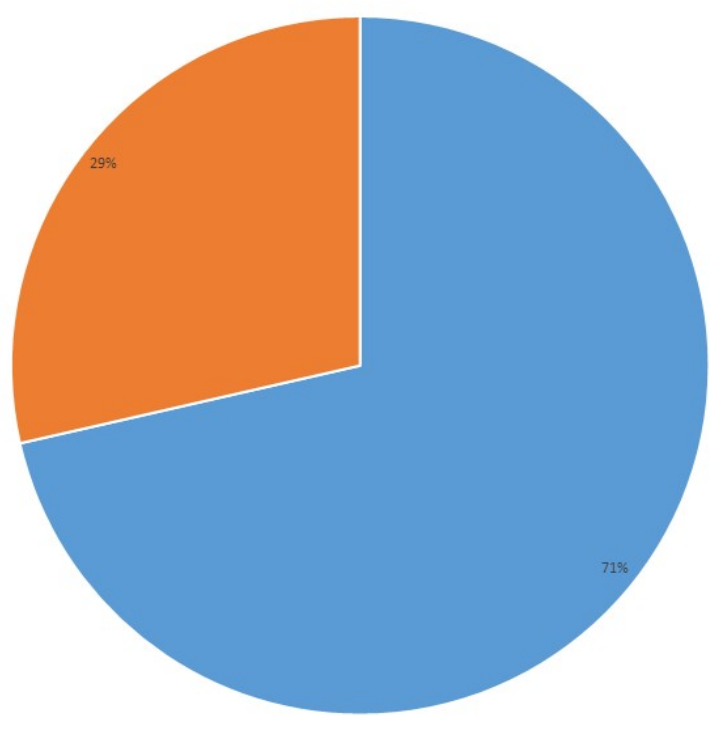

Chart 11. Whether fears can stop respondents from using ultrasound scan services

Most 15 (71.4\%) agreed that this fear can stop them using ultrasound scan services while the least 6 $(28.6 \%)$ reported that fears cannot stop them using ultrasound scan services. 
Table 10. Support received from respondent's partners to access ultrasound scan services

\begin{tabular}{lll}
\hline Support received & Frequency(f) & Percentage (\%) \\
By providing material support & 6 & 50 \\
By providing financial support & 4 & 33.3 \\
Through encouraging and motivating them to attend & 2 & 16.7 \\
Total & $\mathbf{1 2}$ & $\mathbf{1 0 0}$ \\
\hline
\end{tabular}

\section{Figure 12: Whether respondents partner supported them to access ultrasound scan services}

$$
26 \quad n=30
$$

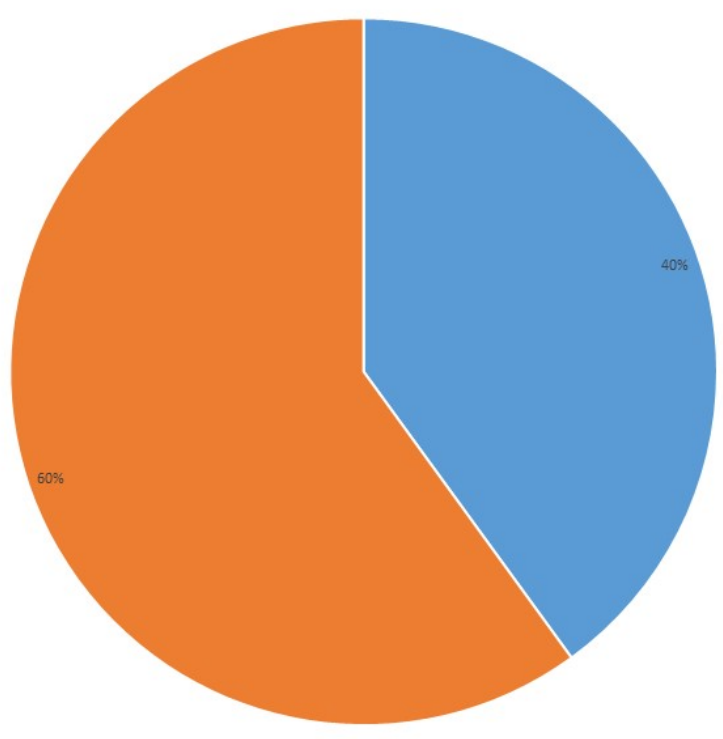

Chart 12. Whether respondents partner supported them to access ultrasound scan services

Study results showed that $18(60 \%)$ respondents reported that their partners did not support them to access ultrasound scan services while the least $12(40 \%)$ reported receiving support.

\section{Table 10: Support received from respondent's partners to access ultrasound scan services $n=12$}

Most 6 (50\%) reported receiving material support, followed by 4 (33.3\%) who reported financial support while the least $2(16.7 \%)$ reported being encouraged and motivated to attend the services. $n=10$

Most $4(40 \%)$ reported lack of awareness about the services as a reason for not using ultrasound scan services, followed by 3 (30\%) who reported fear of using the services, 2 (20\%) reported lack of money to pay for the services while the least $1(10 \%)$ reported lack of interest. 


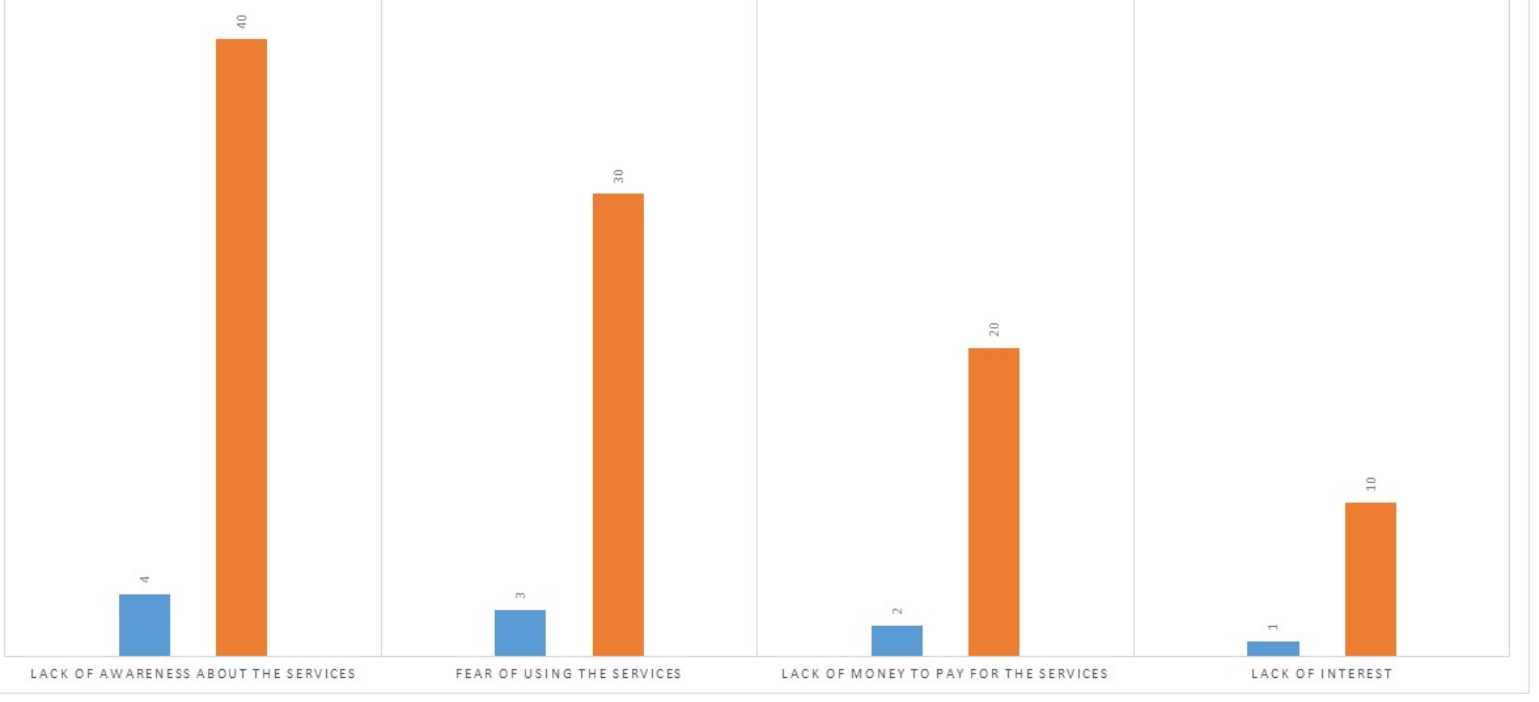

Chart 13. Reasons for not using ultrasound scan services

Table 11. Other socio economic factors influence utilization of ultrasound scan services among pregnant mothers

\begin{tabular}{lll}
\hline Socio economic factors & Frequency(f) & Percentage (\%) \\
Inadequate prenatal care & 6 & 20 \\
Young maternal age & 5 & 16.7 \\
Low education level & 4 & 13.3 \\
Single parenthood & 5 & 16.7 \\
Lack of awareness of the benefits of the ultrasound scan & 4 & 13.3 \\
Long distance to health facilities & 3 & 10 \\
Maternal social status and economic power & 3 & 10 \\
Total & $\mathbf{3 0}$ & $\mathbf{1 0 0}$ \\
\hline
\end{tabular}

\section{Table 11: Other socio economic factors influence utilization of ultrasound scan services among pregnant mothers}

Study results showed that 6 (20\%) respondents reported inadequate prenatal care, followed by $5(16.7 \%)$ who reported young maternal age, $5(16.7 \%)$ reported single parenthood, 4 (13.3\%) reported lack of awareness of the benefits of the ultrasound scan, 4 (13.3\%) reported low education level while the least 3 (10\%) reported long distance to health facilities and $3(10 \%)$ reported maternal social status and economic power.

\subsection{Health Facility Factors Influencing Utilization of Ultrasound Scan Services among Pregnant Mothers}

\section{Figure 14: Whether Ndejje Health Center IV is equipped to provide ultrasound scan services $\quad \mathrm{n}=30$}

Most 20 (66.7\%) respondents reported that Ndejje Health Center IV is not equipped to provide ultrasound scan services while the least 10 (33.3\%) reported that it is equipped to provide ultrasound scan services. 


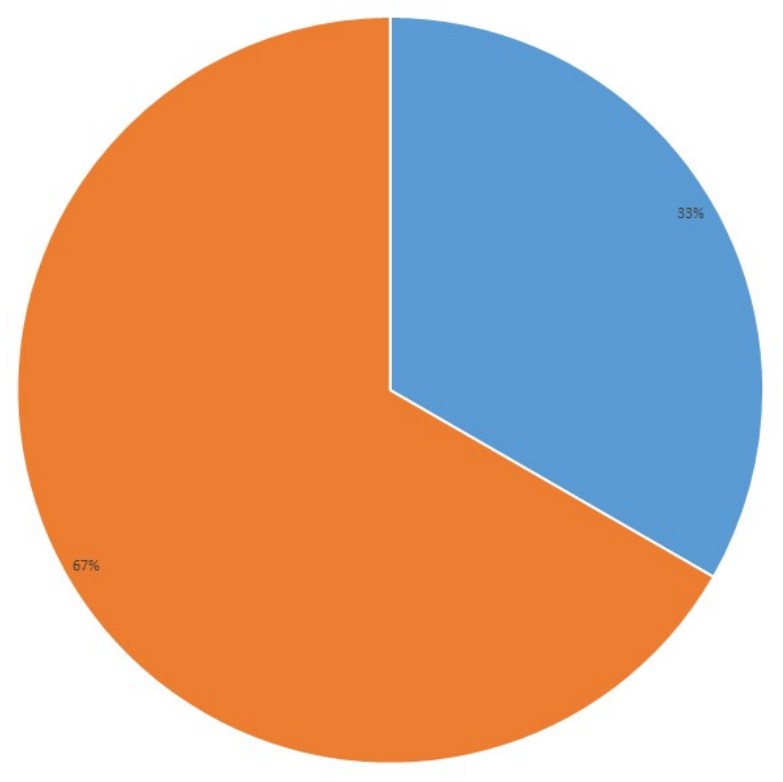

Chart 14. Whether Ndejje Health Center IV is equipped to provide ultrasound scan services

Table 12. Reasons why Ndejje Health Center IV is equipped to provide ultrasound scan services

\begin{tabular}{lll}
\hline Reasons & Frequency(f) & Percentage (\%) \\
Health workers are available & 4 & 40 \\
Health workers are friendly and welcoming & 6 & 60 \\
Total & $\mathbf{1 0}$ & $\mathbf{1 0 0}$ \\
\hline
\end{tabular}

Table 12: Reasons why Ndejje Health Center IV is equipped to provide ultrasound scan services $\mathrm{n}=10$

Most 6 (60\%) reported that health workers were friendly and welcoming while the least 4 (40\%) reported that health workers are available.

Figure 15: Reasons why Ndejje Health Center IV is not equipped to provide ultrasound scan services $\mathrm{n}=20$

Most 12 (60\%) reported frequent breakdown and poor maintenance of equipment, followed by 5 (25\%) reported very long waiting time to receive services while the least $3(15 \%)$ reported that services are not available.

Majority $18(60 \%)$ of respondents reported that ultrasound scan services were not readily available at Ndejje Health Center IV while the least 12 (40\%) reported that ultrasound scan services were readily available at Ndejje Health Center IV.

Most $12(60 \%)$ reported that health workers had positive attitudes during the provision of ultrasound scan services while the least $8(40 \%)$ reported that health workers had negative attitudes.

\section{Table 13: Reasons why respondents thought health workers had positive attitudes $\mathrm{n}=12$}

Most 7 (58.3\%) reported that they were welcoming and friendly while the least 5 (41.7\%) reported that the health workers sensitized them about the importance of ultrasound scan services. 


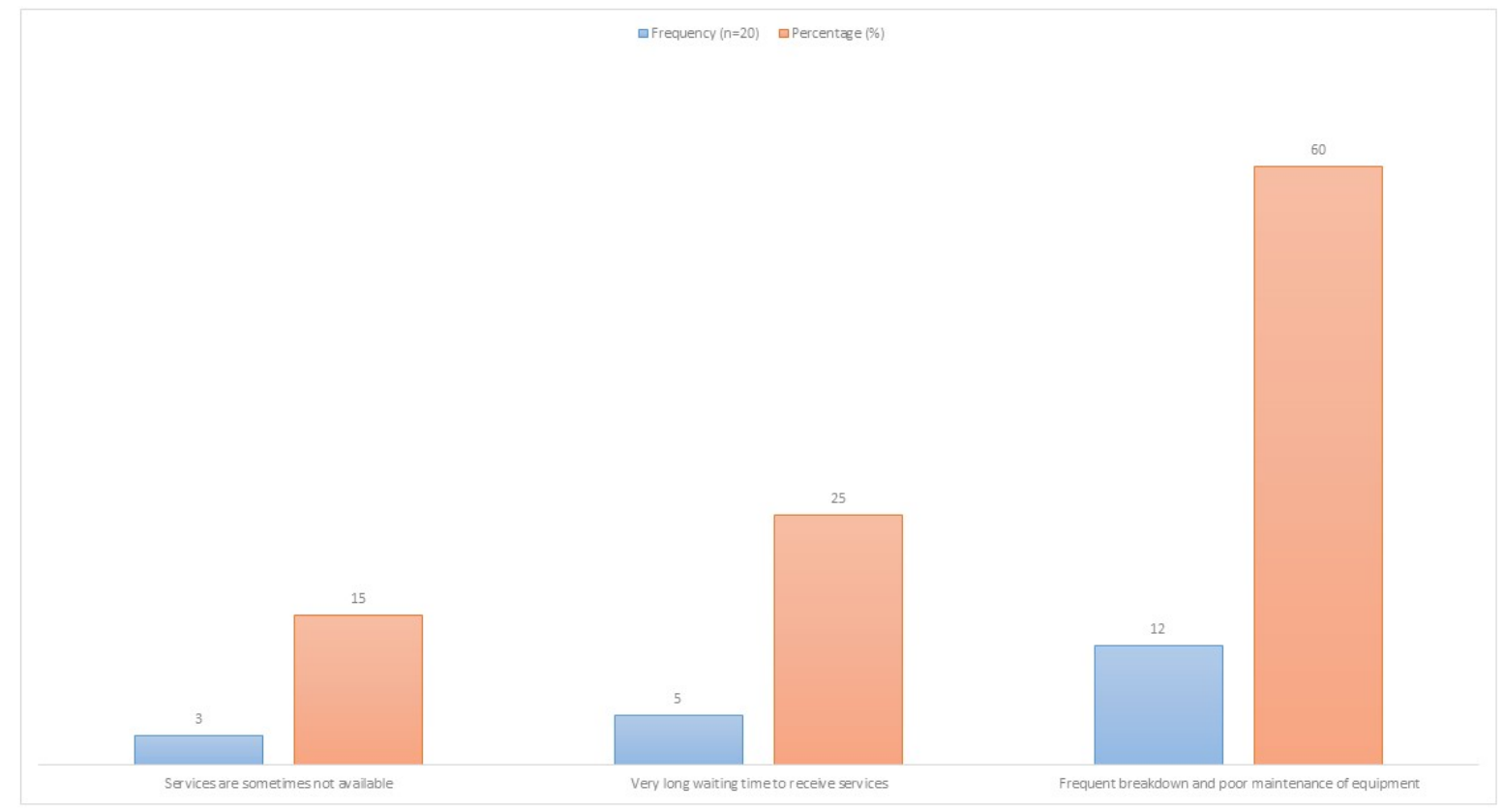

Chart 15. Reasons why Ndejje Health Center IV is not equipped to provide ultrasound scan services

Table 13. Reasons why respondents thought health workers had positive attitudes

\begin{tabular}{lll}
\hline Reasons & Frequency(f) & Percentage (\%) \\
They were welcoming and friendly & 7 & 58.3 \\
They sensitized them about the importance of ultrasound scans & 5 & 41.7 \\
Total & $\mathbf{1 2}$ & $\mathbf{1 0 0}$ \\
\hline
\end{tabular}

Most 12 (60\%) reported that they waited for more than 2 hours to receive services, followed by 5 (25\%) who waited for 40 minutes -1 hour while the least $3(15 \%)$ waited for less than 30 minutes.

\section{Table 14: Health facility factors influencing utilization of ultrasound scan services among pregnant mothers $\quad \mathrm{n}=\mathbf{3 0}$}

Study results showed that $8(26.7 \%)$ respondents reported long waiting time, followed by 7 (23.3\%) who reported provision of poor quality health care services, 5 (16.7\%) reported inefficiency of staff, 4 (13.3\%) reported unavailability of services, $4(13.3 \%)$ reported poor health education and sensitization while the least 2 (6.7\%) reported poor customer care skills among health workers.

Table 14. Health facility factors influencing utilization of ultrasound scan services among pregnant mothers

\begin{tabular}{lll}
\hline Health facility factors & Frequency(f) & Percentage (\%) \\
Poor customer care skills among health workers & 2 & 6.7 \\
Poor health education and sensitization & 4 & 13.3 \\
Provision of poor quality health care services & 7 & 23.3 \\
Long waiting time to receive scanning services & 8 & 26.7 \\
Inefficiency of staff & 5 & 16.7 \\
Unavailability of services & 4 & 13.3 \\
Total & $\mathbf{3 0}$ & $\mathbf{1 0 0}$ \\
\hline
\end{tabular}




\section{Discussion, Conclusions and Recommendations}

\section{Discussion}

Demographic and social characteristics

$15(50 \%)$ of the respondents were in the age range of $26-35$ years. This demonstrated that most respondents were mature individuals which implied that respondents would be more knowledgeable and aware of services such as ultrasound scan services. This study finding was contrary to another study by Gardiner (2017) about advances in fetal echocardiography report that socio-economic factors influencing utilization of ultrasound scan services among pregnant mothers included young maternal age.

$20(66.7 \%)$ of the respondents were married. This implied that they would have financial, physical, and emotional support from their partners/husbands to ensure attendance of antenatal services like utilization of ultrasound scan services. This study finding was in contrast with Mensah et al., (2014), whose study about the Ghanaian woman's experience and perception of ultrasound use in antenatal care that some of the socio-economic factors influencing utilization of ultrasound scan services among pregnant mothers included being unmarried and lack of support among others.

Study results showed that $12(40 \%)$ respondents attained secondary level education, 8 (26.7\%) who attained tertiary level education while most respondents' partners $8(40 \%)$ attained tertiary level education, followed by $5(25 \%)$ attained secondary level education. This implied that since most respondents and their spouses attained a good level of education, they would be more exposed and aware of the importance and benefits of ultrasound scan services during pregnancy. This study finding was opposed by (Barber et al., 2017), whose study found out that socio-economic factors influencing utilization of ultrasound scan services among pregnant mothers included lower education level as well as being a single parent.

Study results showed that $12(40 \%)$ respondents were self-employed while 7 (35\%) respondents' spouses were self-employed. This implied that since most respondents and their spouses were involved in income-generating activities, they would be better able to access and pay for ultrasound scan services as well as pay for transport to the facility. This study finding was in agreement with another study by Heaman et al., 2018 about the inequities in utilization of prenatal care: a population-based study in the Canadian province of Manitoba that some of the socio-economic factors influencing utilization of ultrasound scan services among pregnant mothers included maternal social status and economic power among others. 15 (50\%) of the respondents reported having parity of $2-3$ pregnancies, followed by 10 (33.3\%) who reported a parity of more than 4 pregnancies. This demonstrated that most respondents had a parity of more than 2 pregnancies. This implied that they would be more knowledgeable about self-care during pregnancy as well as the essential need for ultrasound scans during pregnancy.

Socio-Economic Factors Influencing Utilization of Ultrasound Scan Services Among Pregnant Mothers

All had ever heard about ultrasound scan services, and most 20 (66.7\%) respondents obtained information about ultrasound scan services from health workers. This implied that since most respondents had ever heard of ultrasound scan services, they would be better able to make use of this service during pregnancy as it presented many benefits. This study finding was different from Debessai et al., (2016)whose study about inadequate prenatal care use among Canadian mothers: findings from the maternity experiences survey that some of the socio-economic factors influencing utilization of ultrasound scan services among pregnant mothers included lack of awareness of the benefits of the ultrasound scan.

Furthermore, the majority $20(66.7 \%)$ of respondents had ever been health educated about the benefits of using ultrasound scan services including $12(60 \%)$ who reported that it helped to assess the health of the foetus while the least $8(40 \%)$ reported that it helped to detect any challenges on the foetus. This implied that since respondents were aware of the benefits of ultrasound scan services, they would endeavour to make full use of the services as recommended to achieve these benefits. This study finding was contrary to Swanson et al., (2014) whose study about the diagnostic impact of limited, screening obstetric ultrasound when performed by midwives in rural Uganda reported that socio-economic factors influencing utilization of ultrasound scan services among pregnant mothers included lack of awareness or knowledge about the services due to low level of education as well as limited access to information. 
The majority of respondents (66.7\%) had ever used ultrasound scan services and out of the 20 respondents who had used ultrasound scan services, most 10 (50\%) had used the ultrasound scan services once, followed by 7 (35\%) who used the services twice. This demonstrated that most respondents had made use of the ultrasound scan services. However, most had only scanned once yet in figure 9, most were already in the 3rd trimester which may not enable them to achieve all the benefits provided by the scan in checking up on the baby's development throughout the pregnancy.

Most $20(66.7 \%)$ respondents reported that the cost of ultrasound scan services was not affordable while 25 (83.3\%) of respondents agreed that the unaffordability of ultrasound scan services affected the utilization of services. This implied that the high cost of the ultrasound scan services could be one of the reasons which limited respondents' use of the services. This study finding was in line with another study by Mbuyita et al, (2015) whose study reported that socio-economic factors influencing utilization of ultrasound scan services among pregnant mothers included the high cost of services as well as poverty among others.

Out of the 20 respondents who had used ultrasound scan services, most $12(60 \%)$ used the services in the 3rd trimester, followed by 5 (25\%) who used the services in the 2 nd trimester while the least $3(15 \%)$ used the services in 1st trimester. This demonstrated that most respondents had used the ultrasound screening services once mostly in the 3rd trimester which implied that since it was used late, it could lead to late identification/isolation and treatment of any anomaly. This study finding was in agreement with Rao and Platt (2016) whose study about ultrasound screening: Status of markers and efficacy of screening for structural abnormalities revealed that socio-economic factors influencing utilization of ultrasound scan services among pregnant mothers included inadequate prenatal care (prenatal care initiated after the 4th month of pregnancy, and having fewer prenatal care visits).

The majority 21 (70\%) of respondents reported having fears about using ultrasound scan services and out of the 21 respondents who feared using the ultrasound scan services, most 14 (66.7\%) reported fears that the scan will identify bad conditions on the baby while the least 7 (33.3\%) reported fears about what it will reveal. This implied that prevalent fears about the ultrasound scan services directly affected the uptake and utilization of ultrasound scan services. This study finding was in line with another study by Gardiner (2017) about advances in fetal echocardiography report that socio-economic factors influencing utilization of ultrasound scan services among pregnant mothers included fears about the results of the scanning.

Out of the 21 respondents who had feared using ultrasound scan services, most 15 (71.4\%) agreed that this fear can stop them from using ultrasound scan services. This demonstrated the negative impact of fears for scans among pregnant mothers and this hence required immediate addressing through regular health education of mothers during ANC. This study finding was in line with another study by Barber et al., (2017), whose study about maternal body mass index and access to antenatal care: a retrospective analysis of 619,502 births in England revealed that socio-economic factors influencing utilization of ultrasound scan services among pregnant mothers included fear of what the scan would reveal.

Study results showed that $18(60 \%)$ respondents reported that their partners did not support them to access ultrasound scan services. This implied that most pregnant mothers failed to adequately utilize ultrasound scans due to a lack of support from their spouses/partners and especially since most respondents 20 (67\%) considered the service unaffordable. This study finding was in line with Mensah et al., (2014) whose study about the Ghanaian woman's experience and perception of ultrasound use in antenatal care reported that some of the socio-economic factors influencing utilization of ultrasound scan services among pregnant mothers included lack of support among others.

Health Facility Factors Influencing Utilization of Ultrasound Scan Services Among Pregnant Mothers

Most respondents 20 (66.7\%) respondents reported that Ndejje Health Centre IV is not equipped to provide ultrasound scan services due to $12(60 \%)$ frequent breakdown and poor maintenance of equipment. This demonstrated that the provision of quality ultrasound scan services was affected by the frequent breakdown and poor maintenance of equipment which ultimately affected service availability and utilization. This study finding was in line with Dias et al., (2014) whose study about ultrasound diagnosis of foetal renal abnormalities revealed that health facility factors influencing utilization of ultrasound scan 
services among pregnant mothers included unavailability of services as well as understaffing, which led to long waiting to receive services.

However, out of 10 respondents who reported that Ndejje Health Centre IV is equipped to provide ultrasound scan services, most $6(60 \%)$ reported that health workers were friendly and welcoming while the least $4(40 \%)$ reported that health workers are available. This implied that health workers sometimes endeavoured to ensure the good provision of ultrasound scan services by ensuring they were available and providing services with good customer care services. This study finding was contrary to Dibaba et al., (2013), whose study about the effects of pregnancy intention on the use of antenatal care services: systematic review and meta-analysis reported that health facility factors influencing utilization of ultrasound scan services among pregnant mothers included poor customer care skills among health workers.

The majority of respondents (60\%) reported that ultrasound scan services were not readily available. This implied that the unavailability of services could be attributed to frequent breakdown and poor maintenance of the equipment which ultimately led to poor utilization of the services by mothers. This study finding was in agreement with Doubilet (2014) whose study about ultrasound evaluation of the first trimester also revealed that health facility factors influencing utilization of ultrasound scan services among pregnant mothers included unavailability of services as well as the provision of poor quality ultrasound scanning services.

Most respondents $12(60 \%)$ reported that health workers had positive attitudes during the provision of ultrasound scan services which implied that this could contribute to better utilization of ultrasound scan services. This study finding contrasted with the findings of Dibaba et al., (2013), whose study about the effects of pregnancy intention on the use of antenatal care services: systematic review and meta-analysis reported that health facility factors influencing utilization of ultrasound scan services among pregnant mothers included poor customer care skills among health workers.

Most 7 (58.3\%) respondents reported that they were welcoming and friendly while the least $5(41.7 \%)$ reported that the health workers sensitized them about the importance of ultrasound scan services. This demonstrated that some health workers had good practices which could positively influence service and uptake and utilization. This study finding was opposed by the findings of Hawley et al., (2013), whose study about the barriers to adequate prenatal care utilization in American Samoa revealed that health facility factors influencing utilization of ultrasound scan services among pregnant mothers included poor health education and sensitization about the availability and benefits of ultrasound scan services.

Most $12(60 \%)$ respondents reported that they waited more than 2 hours to receive services, followed by 5 (25\%) who waited for 40 minutes -1 hour. This demonstrated that most respondents who used the ultrasound scan services had to wait a considerable amount of time for them to be able to utilize the services and this could put them off using the services as they could not bear waiting for long periods due to their condition. This study finding was in agreement with Wylie et al., (2013), whose study about gestational age assessment in malaria pregnancy cohorts: a prospective ultrasound demonstration project in Malawi reported that poor coverage of ultrasound scanning services especially in rural areas as well as inefficiency and long waiting time to receive services are some of the health facility factors influencing utilization of ultrasound scan services among pregnant mothers.

Study results showed that $8(26.7 \%)$ respondents reported long waiting time to receive scanning services as a health facility factor influencing utilization of ultrasound scan services among pregnant mothers which was supported by Chiavarini et al.,(2015), whose study about immigrant mothers and access to prenatal care: evidence from a regional population study in Italy revealed that health facility factors influencing utilization of ultrasound scan services among pregnant mothers included long waiting time to receive scanning services which ultimately led to poor turn up for services.

\section{Conclusion}

The study revealed various socio-economic factors influencing the utilization of ultrasound scan services among pregnant mothers. For example, although all had ever heard about ultrasound scan services, most had ever used ultrasound scan services once in the 3rd trimester. This was attributed to various factors 
including unaffordability of ultrasound scan services, having fears about using ultrasound scan services including the fear that the scan would identify bad conditions on the baby as well as lack of partner support. The study also revealed that respondents faced various health facility factors which influenced their utilization of ultrasound scan services. For example, most respondents reported that Ndejje Health Centre IV was not equipped to provide ultrasound scan services due to frequent breakdown and poor maintenance of equipment which led to ultrasound scan services not being readily available as well as long waiting time to receive services as most waited more than 2 hours to receive services.

\section{Recommendations}

The Ministry of Health should strengthen the available national sensitization programs aimed at encouraging pregnant women to ensure adequate use of prenatal care during which emphasis should be placed on using services such as ultrasound scan services.

There is also a need for the government to assist health facilities to acquire quality equipment to enable the adequate provision of ultrasound scan services and ready availability of services at times.

The administration of Ndejje Health Centre IV should seek to improve its staffing levels as well as acquire more equipment to reduce waiting time for services.

Regular continuous professional development programs should be provided to staff on how to improve efficiency during the provision of health services as a way to encourage more clients to take up the services.

\section{Implications for the nursing practice}

Health workers at Ndejje Health Centre IV and in other facilities have an important role to play in improving the use of ultrasound scan services by pregnant women. This can be achieved through regular health education of pregnant women about the need and importance of using ultrasound scan services.

\section{Limitation of the Study}

The researcher encountered financial constraints in gathering information from the internet and libraries and printing costs. The researcher overcame this limitation by drawing up a budget which was strictly followed to utilize the available resources.

The researcher also encountered time constraints in the course of the study, balancing the research study and other demanding course works. The researcher overcame these limitations by drawing up a timetable that was strictly followed to overcome the time barriers.

portable ultrasound devices in low- and middle-income countries: a systematic review of the literature. Tropical Med Int Health. 21:294-311.

\section{Reference}

1) Abdullah, P., Kurtz, C.L., McCague, H., Macpherson, A., \&Hala, T. (2019). Factors associated with the timing of the first prenatal ultrasound in Canada. BMC Pregnancy and Childbirth19:164.

2) Barber, C., Rankin, J., \& Heslehurst, N. (2017). Maternal body mass index and access to antenatal care: a retrospective analysis of 619,502 births in England. BMC Pregnancy Childbirth. 17;17:290.

3) Becker, D.M., Tafoya, C.A., Becker, S.L., Kruger, G.H., \&Tafoya, M.J. (2016). The use of portable ultrasound devices in low- and middle-income countries: a systematic review of the literature. Tropical Med Int Health. 21:294-311.

4) Blask, A.N., \&Fagen, K. (2016). Prenatal imaging of the gastrointestinal tract with postnatal imaging correlation. Ultrasound Q.;32:15-24.

5) Chiavarini, M., Lanari, D., Minelli, L., Pieroni, L., \&Salmasi, L. (2016). Immigrant mothers and access to prenatal care: evidence from a regional population study in Italy. BMJ Open.;6 (08):02 - 18.

6) Debessai, Y., Costanian, C., Roy, M., El-Sayed, M., \&Tamim, H. (2016). Inadequate prenatal care use among Canadian mothers: findings from the maternity experiences survey. J Perinatol.36:420-6.

7) Dibaba, Y., Fantahun, M., \&Hindin, M.J. (2013). The effects of pregnancy intention on the use of antenatal care services: systematic review and meta-analysis. Reprod Health.10:50.

8) Gardiner, H.M. (2017). Advances in fetal echocardiography.Semin Fetal Neonatal Med.;23(2):112-8. 
9) Hawley, N.L., Brown, C., Nu'usolia, O., Ah-Ching, J., \&Muasau-Howard, B.(2014). Barriers to adequate prenatal care utilization in American Samoa. Matern Child Health J. 18:2284-92.

10) Heaman, M.I., Martens, P.J., Brownell, M.D., Chartier, M.J., Thiessen, K.R., \&Derksen, S.A.(2018). Inequities in utilization of prenatal care: a population-based study in the Canadian province of Manitoba.BMC Pregnancy Childbirth.18:430.

11) Heaman, M.I., Moffatt, M., Elliott, L., Sword, W., Helewa, M.E., \&Morris, H. (2014). Barriers, motivators and facilitators related to prenatal care utilization among inner-city women in Winnipeg, Canada: a casecontrol study. BMC Pregnancy Childbirth. 14:227.

12) Huang, K., Tao, F., Raven, J., Liu, L., Wu, X., \&Tang, S. (2012). Utilization of antenatal Ultrasound scan and implications for caesarean section: a cross-sectional study in rural Eastern China. BMC Health Services Research12: 93.

13) Mbuyita, S., Tillya, R., Kinyonge, I., \&Shaban, J. (2015). Effects of introducing routinely ultrasound scanning during ante Natal Care (ANC) clinics on number of visits of ANC and facility delivery: a cohort study. Arch Public Health.73:36.

14) Mcclure, E., Goldenberg, R., Swanson, D., Saleem, S., \&Esamai, F. (2017). Routine antenatal ultrasound in low/middle income countries: a cluster randomized trial. Am J ObstetGynecol Oral Plenary Session I.

15) Mensah, Y.B., Nkyekyer, K., \&Mensah, K. (2014). The Ghanaian woman's experience and perception of ultrasound use in antenatal care. Ghana Med J. 48:31.

16) Oluoch, D.A., Mwangome, N., Kemp, B., Seale, A.C., \&Koech, A. (2015). You cannot know if it's a baby or not a baby': uptake, provision and perceptions of antenatal care and routine antenatal ultrasound scanning in rural Kenya. BMC Pregnancy Childbirth.15:127.

17) Papageorghiou, A.T., Kemp, B., Stones, W., Ohuma, E.O., Kennedy, S.H., \&Purwar, M. (2016). Ultrasound-based gestational-age estimation in late pregnancy.UltrasoundObstet Gynecol. 48:719-26.

18) Swanson, D., Lokangaka, A., Bauserman, M., Swanson, J., \&Nathan, R.O. (2017). Challenges of implementing antenatal ultrasound screening in a rural study site: a case study from the Democratic Republic of the Congo. Global health, science and practice.5:315-24

19) Swanson, J.O., Kawooya, M.G., Swanson, D.L., Hippe, D.S., \&Dungu-Matovu, P. (2014). The diagnostic impact of limited, screening obstetric ultrasound when performed by midwives in rural Uganda. J Perinatol.34:508-12.

20) Wylie, B.J., Kalilani-Phiri, L., Madanitsa, M., Membe, G., \&Nyirenda, O. (2013). Gestational age assessment in malaria pregnancy cohorts: a prospective ultrasound demonstration project in Malawi. Malar J.12:183. 